\title{
Understanding the mHealth implementation and adoption impact: A FVM perspective
}

\author{
Nilmini Wickramasinghe \\ Deakin University, Faculty of Health
}

\begin{abstract}
Successful adoption and implementation of Mobile Health applications (mHealth) has been the subject of extensive research as can be evidenced by the large body of research literature on this topic. However, the fit and viability perspective issues have been less widely studied, perhaps because of the difficulty and complexity of healthcare services and delivery factors and their studies. In today's global business environment this remains a key issue and critical success factor. Hence, this paper explains how challenging the mHealth application implementation success is for organizations government and patients. Furthermore, it is also very important to assess a fit and viability of mHealth application implementations in diverse environment. By drawing upon multiple streams of theory building, a framework, from the Fit-Viability Model (FVM) perspective, is developed. The framework provides important and valuable guiding principles for the decisions on adoption and deployment of mHealth applications. Grounded on the fit-viability framework, the paper advocates the use of a multi-case study approach to examine the success or failure of mHealth applications adoption and implementation. Managers and researchers can use the developed framework to identify the fit and viability of mHealth applications implementations to increase the possibility of success.
\end{abstract}

Keywords: mHealth applications implementation; Fit-Viability Model; mHealth applications implementation

\section{Introduction}

Mobile devices are changing the way healthcare is delivered. Mobile devices are offering mobility, flexibility, convenience and real-time communication in healthcare ${ }^{[1-4]}$. The ability to monitor patients' health remotely is making mobile devices' popular in health domain Patients are using mobile devices for consultation with doctors ${ }^{[5]}$. On the other hand, doctors are using mobile devices in collaboration with other technologies (like skype, FaceTime and internet technology) for real time monitoring/tele monitoring of patients ${ }^{[6,7]}$.

The concept of use of mobile devices in healthcare can be viewed as follows: any equipment (having different sensors) which can be worn as wrist band, implanted in the body or embedded with the living spaces, measuring different physical changes of the patients, monitoring physical activities, analyzing, alerting and communicating with healthcare professionals and patients from remote places ${ }^{[8]}$. Use of mobile devices in healthcare is benefiting healthcare domain in many ways such as reducing cost and traveling time for patients, cares and specialists. Hence, the concept of using mobile devices in healthcare is more beneficial then tradition healthcare services where patient have to wait in long queues and doctors are overburdened.

Use of mobile devices in healthcare has enhanced the scope of health care services and in the near future will make health services more flexible ${ }^{[1,2,9]}$. For example, ${ }^{[10]}$ proposes an ensemble sensing network named w-health (wireless

This is an open-access article distributed under the terms of the Creative Commons Attribution Unported License

(http://creativecommons.org/licenses/by-nc/4.0/), which permits unrestricted use, distribution, and reproduction in any medium, provided the original work is properly cited. 
health) which can combine smart phone, smart watch and smart glass in one network and can be used in tele health in near future.

However, healthcare professionals (HCPs) are reluctant for adopting mobile devices ${ }^{[6,11-13]}$. Many nurses are resistant to the idea of using new technology such as mobile phone in patients care ${ }^{[10,14]}$. According to WHO global observatory for eHealth report by ${ }^{[15]}$ the worldwide adoption rate of mHealth is only $30 \%$. Therefore, it is important to design a conceptual framework for adoption of mobile devices in healthcare domain.

mHealth application's implementation is a difficult and complex decision, system implementation doesn't mean installing software it is much more than a technology adoption. Rather it involves people issues more than technological issues. Research indicates that people issues are more to blame for the unsuccessful efforts of mHealth applications implementations ${ }^{[16]}$. A recent report (www.CIO.com) indicates a citation from an analyst "no major software implementation is really about the software, it's about change management". Thus, the emphasises is that when you move to technology, you are changing the way people work. You are challenging their principles, their beliefs and the way they have done things for many, many years.

mHealth applications implementation problems are strategic, organizational and technical ${ }^{[17]}$. mHealth applications implementation is integrally connected to organizations strategic decisions which in turn are designed to gain a competitive advantage.

Despite the significance of the topic of mHealth applications implementations, very little empirical research has been conducted in this domain. Studies have indicated that culture play a very important role in the process but fail to pinpoint how it affects important and inimitable IT innovations ${ }^{[18,19]}$. One reason for this could be due to the lack of robust and rich models.

By extending the Fit-Viability Model of ${ }^{[20]}$ we have developed a framework to bridge the research gap by considering the impact of national factors on organizational factors in mHealth applications implementations by conceptualizing the main constraints of system fit for the organization. Then we will evaluate the model and the national factors as well as organizational factors on both strategic and tactical levels for successful adoption and implementation of mHealth applications. Soh, Kien and Tay-Yap ${ }^{[21]}$ had identified that system functionality needs to fit with the adopting organization's business processes. Fit here is measured by identifying the requirements of adopting organizations with the technology. This fit would be characterized by identifying the tasks an organization wants to perform with technology and how better it fits with the organization's business processes. Fit would be in the form of a cross-module integration including data standardizations, reporting requirements as well as human resource practices. System success is measured by system performance and system performance will be measured by system use and user satisfaction. Viability here depends on organizational as well as national factors such as political and social, economic, environmental infrastructure/technology and organization factors at a strategic level (leadership, management style, and polices), tactical level (information sharing, training and learning, technical staff, user behavior).

\section{Literature Review}

The key relevant areas in the literature are briefly summarized below.

\subsection{Mobile Health Implementations}

Use of mobile devices in healthcare is a new concept ${ }^{[8]}$. There is not much discussed relating to factors influencing adoption of mobile devices in healthcare. Factors influencing adoption of mobile devices are both at hospital level and individual level (healthcare professional level and patients ${ }^{[10,15,22-25]}$. For example, Wu et al. ${ }^{[25]}$ explore organizations, technology, system services, and individual psychological factors influencing individual intention for adoption of mobile devices. In a survey conducted by Shareef et al..$^{[22]}$ explore perceived ease of use, perceived usefulness, security and privacy and reliability factors influencing adoption of mobile devices in healthcare.

Due to newness of adoption of mobile devices in healthcare there is scarcity of literature in this context. Therefore, researcher has attempted to synthesize literature, for understanding trend for adoption of various types of technology in 
healthcare from HCPs perspective. Bradford et al. ${ }^{[26]}$ studied adoption of tele health technology from healthcare professional perspective and concluded that major factors for adoption are technology, individual and service. Ben-Zeev et al. (1) developed a smartphone system for self-management of Schizophrenia named FOCUS and (during first testing of system) found that individuals were facing problems for using mobile devices in healthcare. The problems are: with abbreviations, large amount of text, difficult wording, font size, smaller touch space, small diameter of the buttons and smaller touch sensitivity. This means mobile devices used should have favoring features with healthcare domain which healthcare professional can easily understand and insert mHealth application. Heidarian and Mason ${ }^{[27]}$ explored factors: technology (technology upgrades), and organization (cost, lack of time for implementation, and training) which are technology and organizational perspective. Various other authors have also mentioned factors for adoption of technology form organizational, technology and individual perspectives ${ }^{[28-32]}$. However, the research on adoption of mobile devices at HCPs level focusing on individual characteristics perspective is limited in literature. Therefore, a conceptual framework is designed to discuss the influence of individual characteristics of HCPs for adoption of mobile devices in healthcare domain.

Several researchers have used various theories and models (Technology Acceptance model, Theory of Reasoned Action, Theory of Planned Behavior, Diffusion of Innovation Theory, Unified Theory of Technology Acceptance and Utilization) to understand adoption behavior for technology in healthcare domain ${ }^{[8,33-37]}$. Theory of planned behavior (TPB) and diffusion of innovation theory (DOI) are used as an underlying theory for developing conceptual framework. In development of conceptual framework, researcher has to understand two dimensions: HCPs and mobile devices to investigate factors influencing HCPs for adoption mobile devices. As TPB have potential to predict the fundamental predictors of behavior while DOI has the potential to explain operational aspect of technology in clinical processes ${ }^{[38]}$. Therefore, both the TPB and DOI have potential to explain factors according to the nature of research.

The basis for mHealth applications implementation and adoption varies from organisation to organisation depending on their need and perceived benefits. There could be many motivational forces for implementation including; one standardized IT platform, data integrity and visibility, better customer care, efficient business processes, better decision making, lack of support for old legacy systems, better return on investment, less operational costs, integration of different systems and platforms and better patient care ${ }^{[17,39-42]}$. mHealth applications implementation is not only a technological initiative but also considered to be a business project because it involves developing a business case, project planning, software configuration and customization, and business process engineering ${ }^{[43]}$.

Although mHealth applications are very promising; there are reports of difficulties and high failure rates. Most of the failures are due to management, social, cultural and organizational issues but not so much on technical aspects. Researchers like ${ }^{[13,42,44-49]}$ have argued that successful implementations require better understanding of cultural differences because of its significance and impact.

Studies done by ${ }^{[16,17,39-42,50-52]}$ somehow successfully recognized many problems linked with mHealth applications implementations. The situation in Asian countries is even more complex and critical ${ }^{[53]}$. According to Sun et al.$^{[33]}$ the success rate in China is less than $10 \%$ as compared to USA where the success rate is $51 \%$ which itself is not very promising. Asian people have different beliefs in sharing information. Employees working in the authoritative environment, like to be told about their day-to-day activities. Organizational processes are more complex and hard to reengineer, processing procedures are complex and incompatible. Information content and data, presentation compatibility is another critical issue $\mathrm{e}^{[54]}$.

For the measurement of success many authors have used the Critical Success Factors (CSFs) approach and have identified system fit with in the organizations business process and viability of system implementation and adoption as a very important success factor ${ }^{[5-61]}$. This approach has its roots in the work of Pinto and Slevin ${ }^{[62]}$. For instance, Esteves and Pastor ${ }^{[5]}$ developed a unified Critical Success Factors Model; Holland and Light ${ }^{[59]}$ suggested a model by dividing CSFs into two groups of strategic and tactical factors; and Al Mashari et al. ${ }^{[57]}$ and Kumar et al. ${ }^{[63]}$ argued that firms can realize quick performance benefits and smoother implementations if they accentuate and stress on CSFs throughout 
their mHealth applications deployment.

The success or failure of any mHealth applications implementation depends on how the mHealth applications are perceived, treated and integrated. When mHealth applications developed in western countries are implemented in eastern countries with quite a different culture, implementation may need to be localized for a better chance of success. On the other hand, companies can potentially lose all or some of the strategic benefits of mHealth applications by localizing them. The problems to transfer mHealth applications to developing countries range from mismatch with the local culture, economic and regulatory requirements to under or over estimation of perceived benefits of mHealth applications implementation ${ }^{[64]}$. Due to these factors companies thus end up having undesirable designs and reality gaps, which could lead to underperforming mHealth applications and eHealth systems ${ }^{[65,66]}$.

\subsection{Fit-Viability Model}

$\operatorname{Tjan}^{[67]}$ proposed fit viability dimensions for evaluating Internet initiative projects. Liang and Liang et al. ${ }^{[20]}$ by taking these two dimensions and adding Task Technology Fit (TTF) theory proposed a fit-viability model to study m-commerce applications. In their framework, viability measures the readiness of the organization for the technology adoption and implementation, and fit measures capabilities of the systems to optimally perform the required tasks. These two dimensions make a simple matrix with fit on horizontal and viability on vertical axis, as shown in figure 1.

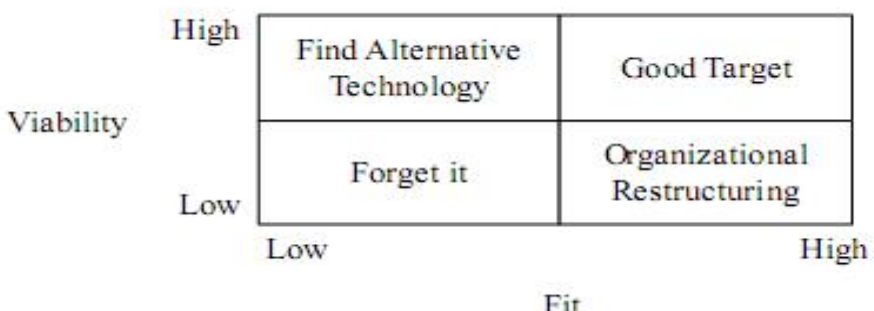

By using the

Figure 1. FIT-Viability Framework (Liang and Wei 2004)

technology adoption and implementation. For example, developing countries could have better political stability, growing economy, better environment, and more stable telecommunication and information technology infrastructure, which mean the system could rate high on viability, but the task does not fit the nature of a mHealth applications would lead to unsuccessful projects. For example, for a mHealth system implemented in two different geographical locations such as USA and China, a system could have high viability and high fit in the USA, but high viability and low fit in China because of different data structure, reporting procedures and business processes which means the system has to be customized according to the local organizational and cultural requirements or the business has to go through organizational restructuring or Business Process Re-engineering (BPR), which could lead to complications, delays and budget overruns. Only those systems with high task-technology fit and high viability are good targets.

\subsection{Task-Technology Fit}

The theoretical basis of the fit construct is derived from the Task-Technology Fit model which according to Goodhue ${ }^{[68,69]}$ argues that a fit between task characteristics and system features need to be high for the better performance and success and this will have effect on the decision-making process of an organization. Research ${ }^{[21,69,70]}$ has indicated that if a system is more aligned with the requirements of the users there are greater chances of system success which leads to better performance. It means that if the features offered by the system fit with the task requirements the users will be more incline to use it.

\subsection{Viability}

Viability refers to the degree of impact of national and organizational factors on a system adoption and implementation decision. These factors at the national level include political and social, economic, environmental as well as infrastructure/technology factors. At the organizational level literature has proposed many factors at the strategic 
and tactical levels ${ }^{[58,71]}$. These factors include leadership, management style, polices, information sharing, training and learning, technical staff, and user behavior. Taking the example of China, Huang and Palvia ${ }^{\text {[72] }}$ suggest that economic and technological factors are crucial factors in mHealth applications implementations; and ignoring these factors could lead to unsuccessful projects. Molla and Loukis ${ }^{[64]}$ argued that stable economic growth and strong IT infrastructure could create better business environments and innovation adoption, positively affecting viability of the system.

\section{Research Framework}

The research framework shown in figure 2 illustrates the key constructs and factors affecting mHealth applications implementations. Fit will be measured by matching the requirements of the organization with the functionalities offered by the system e.g. data format, operating procedures, and output format. Viability will be measured by assessing the impact of national and organizational factors on the adoption decision of the organization.

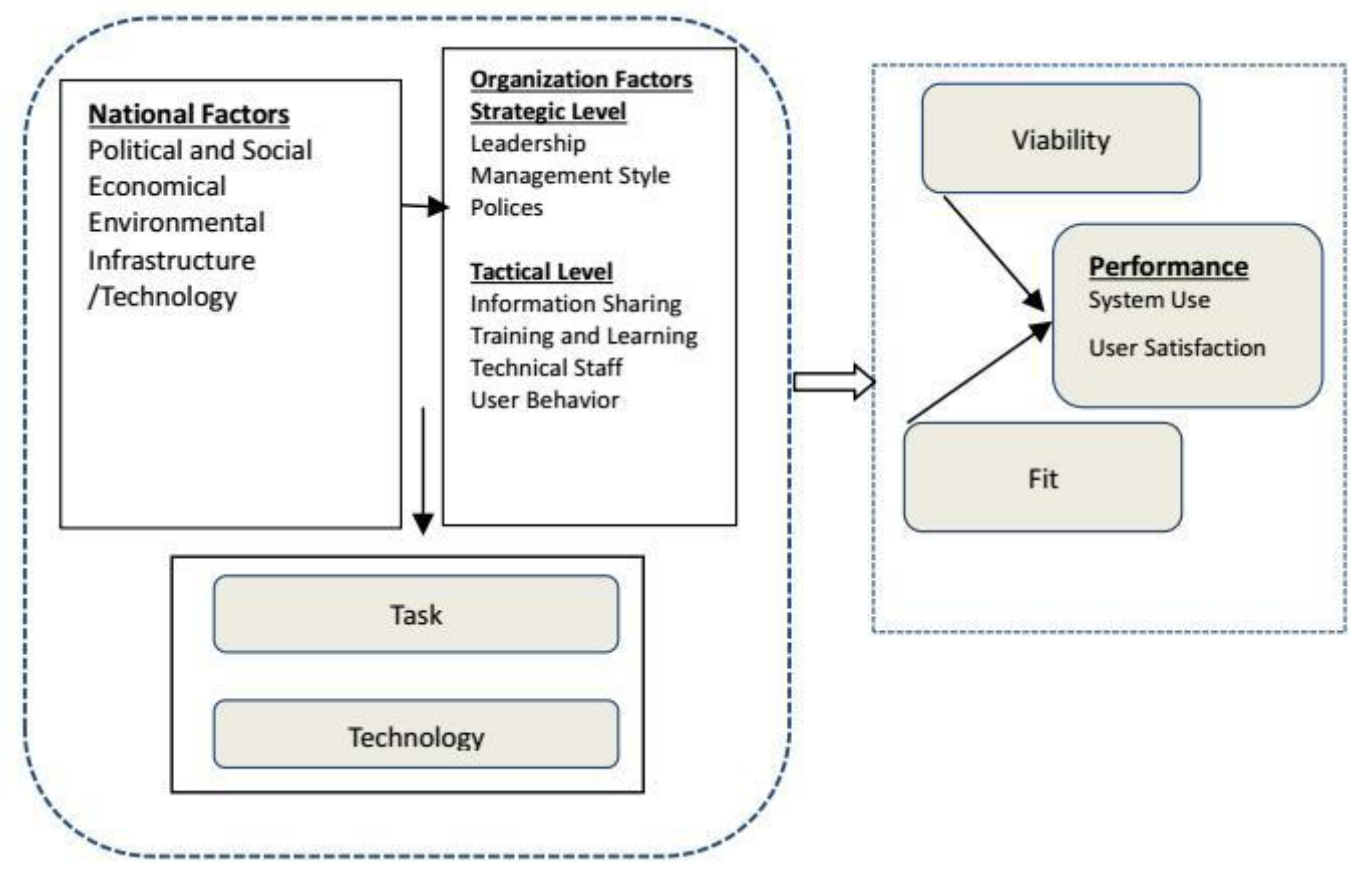

Figure 2. Research Framework

\section{Methodology}

Based on the criteria given by Yin ${ }^{[73]}$; the appropriate choice of methodology to test the use and usability of the proposed framework (figure 2) is a qualitative Multi-case study research because this is an exploratory study of a new phenomenon of mHealth applications implementation. Further, we wish to explore that how an mHealth applications solution in different countries and in different geographical locations can be implemented successfully and what are the factors that impact on the implementation and adoption of these IS based interventions. Qualitative research is holistic, humanistic, and interactive, it can provide more support to focus on the study of a complex phenomenon of human and system interaction and relationship; as in our case mHealth applications implementations ${ }^{[61,73]}$. Qualitative research can provide deeper understanding of the phenomenon as compared to quantitative study because of the exploratory nature of the study and focus which would not be on quantitative measures ${ }^{[74]}$. The chosen case studies are from Australia, China, Germany and USA.

Data will be collected by employing variety of data collection techniques. This will include in-depth, open-ended unstructured interviews with key informants from the respective group of different key stake holders in mHealth applications implementation projects in the chosen countries and regens. These stake holders will include project and IT managers as well as mHealth applications service providers. Standard techniques of qualitative analysis such as coding 
and thematic analysis will be employed ${ }^{[75,76]}$.

\section{Discussion and Conclusion}

The purpose of this research in progress paper is to conceptualize a framework to investigate mHealth applications implementation and adoption, since we recognize that with e-innovations not only the technology solution is necessary but it is also critical to look at organizational and societal aspects concurrently especially in today's global business environment. mHealth applications implementations comprise of technology as well as human involvement. In view of this it is important to investigate the impact of political and social, economic, environmental and infrastructure/technology factors on the organizational decision making. We propose the importance of studying the system viability and fit before making any decision about system adoption and deployment. Technology needs to have a good fit to perform the required tasks, then we anticipate that system viability and fit should have positive effect on the organization's performance and that can be measured by system use and user satisfaction.

Based on this conceptual paper, we plan to pursue a multi-country study, in particular focusing on Australia, china, Germany and the USA, in order to test the use and usability of our proposed framework. Given the large uptake of mHealth applications in all these countries in addition to the fact that health consumers of these countries are spread vastly in different geographic and social classes, we are confident that such a study will serve to provide us with insightful data and key results that will in turn serve to provide the necessary empirical evidence to support the proposed framework. Clearly, until such empirical data can be provided any proposed framework remains theoretical and its practical relevance can be questioned. This limitation with our current conceptualization, while out of scope for this present paper, will be addressed in our follow up future work. We have outlined the research methodology and research design we will follow and plan to commence on the receipt of ethics clearance. The current paper serves to identify the key challenge of mHealth applications implementation and adoption and present the proposed integrative framework as a contender to address the key problems of failure of mHealth applications today due to poor or no conceptualisation.

\section{References}

1. Ben-Zeev D, Kaiser SM, Brenner CJ, Begale M, Duffecy J, Mohr DC. Development and Usability Testing of FOCUS: A Smartphone System for Self-Management of Schizophrenia. Psychiatr Rehabil J. 2013 Dec;36(4):289-96.

2. Morón MJ, Luque R, Casilari E. On the Capability of Smartphones to Perform as Communication Gateways in Medical Wireless Personal Area Networks. Sensors. 2014 Jan 2;14(1):575-94.

3. Nah FF-H, Siau K, Sheng H. The Value of Mobile Applications: A Utility Company Study. Commun ACM. 2005 Feb;48(2):85-90.

4. Wells JD, Sarker S, Urbaczewski A, Sarker S. Studying customer evaluations of electronic commerce applications: a review and adaptation of the task-technology fit perspective. In: 36th Annual Hawaii International Conference on System Sciences, 2003 Proceedings of the. 2003. p. 10 pp.-.

5. Korzep K. The future of technology and the effect it may have on replacing human jobs. Technol Health Care Off $\mathrm{J}$ Eur Soc Eng Med. 2010;18(4-5):353-8.

6. Slaper MR, Conkol K. mHealth tools for the pediatric patient-centered medical home. Pediatr Ann. 2014 Feb;43(2):e39-43.

7. Zangbar B, Pandit V, Rhee P, Aziz H, Hashmi A, Friese RS, et al. Smartphone Surgery: How Technology Can Transform Practice. Telemed E-Health. 2014 Apr 2;20(6):590-2.

8. Dwivedi YK, Shareef M, Simintiras A, Lal B, Weerakkody VJP. A generalised adoption model for services: A cross-country comparison of mobile health (m-health). 2016 Jan [cited 2018 Mar 25]; Available from: https://bradscholars.brad.ac.uk/handle/10454/11684

9. Agosti V., Graziano S., Artiaco L., Sorrentino G. Biological mechanisms of stroke prevention by physical activity in type 2 diabetes. Acta Neurol Scand. 2008 Aug 11;119(4):213-23.

10. Rana R, Hume M, Reilly J, Jurdak R, Soar J. Opportunistic and Context-aware Affect Sensing on Smartphones: The Concept, Challenges and Opportunities. ArXiv150202796 Cs [Internet]. 2015 Feb 10 [cited 2018 Mar 25]; Available from: http://arxiv.org/abs/1502.02796

11. Fox S. The Social Life of Health Information, 2011 [Internet]. Pew Research Center: Internet, Science \& Tech. 2011 [cited 2018 Mar 25]. Available from: 
http://www.pewinternet.org/2011/05/12/the-social-life-of-health-information-2011/

12. Milward J, Day E, Wadsworth E, Strang J, Lynskey M. Mobile phone ownership, usage and readiness to use by patients in drug treatment. Drug Alcohol Depend. 2015 Jan 1;146:111-5.

13. Wu J-H, Wang S-C, Lin L-M. Mobile computing acceptance factors in the healthcare industry: A structural equation model. Int J Med Inf. 2007 Jan;76(1):66-77.

14. Blakemore S-J. Imaging brain development: the adolescent brain. NeuroImage. 2012 Jun;61(2):397-406.

15. Kay M. mHealth: New horizons for health through mobile technologies [Internet]. Cape Town: World Health Organization Global Observatory for eHealth; 2011 Jun p. 21. Available from: http://www.who.int/ehealth/mhealth_summit.pdf

16. Lewis TL, Wyatt JC. mHealth and mobile medical apps: a framework to assess risk and promote safer use. J Med Internet Res. 2014;16(9).

17. Aranda-Jan CB, Mohutsiwa-Dibe N, Loukanova S. Systematic review on what works, what does not work and why of implementation of mobile health (mHealth) projects in Africa. BMC Public Health. 2014;14(1):188.

18. Everdingen YM van, Waarts E. The Effect of National Culture on the Adoption of Innovations. Mark Lett. 2003 Oct 1;14(3):217-32.

19. Png IPL, Tan BCY, Wee K-L. Dimensions of national culture and corporate adoption of IT infrastructure. IEEE Trans Eng Manag. 2001 Feb;48(1):36-45.

20. Liang T-P, Huang C-W, Yeh Y-H, Lin B. Adoption of mobile technology in business: a fit - viability model. Ind Manag Data Syst. 2007 Oct 2;107(8):1154 - 69.

21. Soh C, Kien SS, Tay-Yap J. Enterprise Resource Planning: Cultural Fits and Misfits: Is ERP a Universal Solution? Commun ACM. 2000 Apr;43(4):47-51.

22. Shareef MA, Kumar V, Kumar U. Predicting mobile health adoption behaviour: A demand side perspective. J Cust Behav. 2014 Oct 31;13(3):187-205.

23. Brown W, Yen P-Y, Rojas M, Schnall R. Assessment of the Health IT Usability Evaluation Model (Health-ITUEM) for evaluating mobile health (mHealth) technology. J Biomed Inform. 2013 Dec;46(6):1080-7.

24. West CP. A mixed methods sequential explanatory study of the impact of chronic pain on family resilience [Internet] [phd]. James Cook University; 2012 [cited 2018 Mar 25]. Available from: https://researchonline.jcu.edu.au/24720/

25. Wu Z-Y, Lee Y-C, Lai F, Lee H-C, Chung Y. A Secure Authentication Scheme for Telecare Medicine Information Systems. J Med Syst. 2012 Jun 1;36(3):1529-35.

26. Bradford NK, Armfield NR, Young J, Herbert A, Mott C, Smith AC. Principles of a paediatric palliative care consultation can be achieved with home telemedicine. J Telemed Telecare. 2014 Oct 1;20(7):360-4.

27. Heidarian A, Mason D. Health information technology adoption in New Zealand optometric practices. Clin Exp Optom. 2013 Nov;96(6):557-65.

28. Baig AA, Mangione CM, Sorrell-Thompson AL, Miranda JM. A randomized community-based intervention trial comparing faith community nurse referrals to telephone-assisted physician appointments for health fair participants with elevated blood pressure. J Gen Intern Med. 2010 Jul;25(7):701-9.

29. Brewster L, Mountain G, Wessels B, Kelly C, Hawley M. Factors affecting front line staff acceptance of telehealth technologies: a mixed-method systematic review. J Adv Nurs. 2014 Jan;70(1):21-33.

30. Furukawa MF, Raghu TS, Spaulding TJ, Vinze A. Adoption of health information technology for medication safety in U.S. Hospitals, 2006. Health Aff Proj Hope. 2008 Jun;27(3):865-75.

31. Gagnon M-P, Desmartis M, Labrecque M, Car J, Pagliari C, Pluye P, et al. Systematic Review of Factors Influencing the Adoption of Information and Communication Technologies by Healthcare Professionals. J Med Syst. 2010 Mar 30;36(1):241-77.

32. Tiong IC, Hafeez-Baig A, Gururajan R, Soar J. Preliminary investigation to explore perceptions of security issues associated with wireless technology in healthcare in Australia. HIC 2006 Proc Bridg Digit Divide Clin Consum Comput. 2006;

33. Daim TU, Basoglu N, Topacan U. Adoption of health information technologies: the case of a wireless monitor for diabetes and obesity patients. Technol Anal Strateg Manag. 2013;25(8):923-938.

34. Castro D, Miller B, Nager A. Unlocking the potential of physician-to-patient telehealth services. Inf Technol Innov Found. 2014;

35. Deng Z, Mo X, Liu S. Comparison of the middle-aged and older users' adoption of mobile health services in China. Int J Med Inf. 2014 Mar;83(3):210-24.

36. Saad NM, Alias RA, Ismail Z. Initial framework on identifying factors influencing individuals' usage of telehealth. In: 2013 International Conference on Research and Innovation in Information Systems (ICRIIS). 2013. p. 174-9.

37. Sanders C, Rogers A, Bowen R, Bower P, Hirani S, Cartwright M, et al. Exploring barriers to participation and adoption of telehealth and telecare within the Whole System Demonstrator trial: a qualitative study. BMC Health Serv Res. 2012 Jul 26;12:220.

38. Ghodeswar BM, Vaidyanathan J. Organisational adoption of medical technology in healthcare sector. J Serv Res. 
2007;7(2):57.

39. Alexy Betty B., Elnitsky Christine. Rural Mobile Health Unit: Outcomes. Public Health Nurs. 2007 May $5 ; 15(1): 3-11$.

40. Harvey G, Kitson A. Achieving improvement through quality: an evaluation of key factors in the implementation process. J Adv Nurs. 1996;24(1):185-195.

41. Medhanyie AA, Little A, Yebyo H, Spigt M, Tadesse K, Blanco R, et al. Health workers' experiences, barriers, preferences and motivating factors in using mHealth forms in Ethiopia. Hum Resour Health. 2015;13(1):2.

42. Tate EB, Spruijt-Metz D, O’Reilly G, Jordan-Marsh M, Gotsis M, Pentz MA, et al. mHealth approaches to child obesity prevention: successes, unique challenges, and next directions. Transl Behav Med. 2013;3(4):406-415.

43. Markus ML, Tanis C. The enterprise systems experience-from adoption to success. Fram Domains IT Res Glimpsing Future Past. 2000;173:207-173.

44. Applegate LM, McFarlan FW, McKenney JL. Corporate information systems management : the challenges of managing in an information age [Internet]. 5th ed. Boston ; London : Irwin/McGraw-Hill; 1999 [cited 2018 Mar 25]. Available from: https://trove.nla.gov.au/version/39642446

45. McFarland DJ, Cacace AT. Defining perception and cognition. Behav Brain Sci. 1999;22(03):385-385.

46. Harris R, Davison R. Anxiety and involvement: Cultural dimensions of attitudes toward computers in developing societies. In: Global perspective of information technology management. IGI Global; 2002. p. 234-259.

47. Bancroft J. Reply from Dr J. Bancroft. Diabet Med. 2004 Jul 19;13(10):916-916.

48. Burn J, Davison R, Jordan E. The Information Society - A Cultural Fallacy? Fail Lessons Learn Inf Technol Manag. 1997 Dec 1;1(4):219-41.

49. Hofstede G. Dimensionalizing cultures: The Hofstede model in context. Online Read Psychol Cult. 2011;2(1):8.

50. Chib A. The promise and peril of mHealth in developing countries. Mob Media Commun. 2013;1(1):69-75.

51. Manda TD, Sanner TA. Bootstrapping Information Technology Innovations Across Organisational and Geographical Boundaries: Lessons from an mHealth Implementation in Malawi. In: Selected Papers of the Information Systems Research Seminar in Scandinavia: Designing the Interactive Society. 2012.

52. Tomlinson M, Rotheram-Borus MJ, Swartz L, Tsai AC. Scaling up mHealth: where is the evidence? PLoS Med. 2013;10(2):e1001382.

53. Sun J, Guo Y, Wang X, Zeng Q. mHealth For Aging China: Opportunities and Challenges. Aging Dis. 2016 Jan 2;7(1):53-67.

54. Miguel CD, Guo C, Lund H, Feng D, Mattson DL. Infiltrating T lymphocytes in the kidney increase oxidative stress and participate in the development of hypertension and renal disease. Am J Physiol-Ren Physiol. 2010;300(3):F734-F742.

55. Esteves J, Pastor J. Towards the unification of critical success factors for ERP implementations. In: 10th Annual BIT Conference, Manchester, UK. 2000.

56. Parr A, Shanks G. A model of ERP project implementation. J Inf Technol. 2000 Dec 1;15(4):289-303.

57. Al-Mashari M, Al-Mudimigh A, Zairi M. Enterprise resource planning: A taxonomy of critical factors. Eur J Oper Res. 2003;146(2):352-364.

58. Umble EJ, Haft RR, Umble MM. Enterprise resource planning: Implementation procedures and critical success factors. Eur J Oper Res. 2003 Apr 16;146(2):241-57.

59. Holland CR, Light B. A critical success factors model for ERP implementation. IEEE Softw. 1999;16(3):30-36.

60. Tatsiopoulos IP, Panayiotou NA, Kirytopoulos K, Tsitsiriggos K. Risk management as a strategic issue for the implementation of ERP systems: a case study from the oil industry. Int J Risk Assess Manag. 2003;4(1):20-35.

61. Motwani J, Subramanian R, Gopalakrishna P. Critical factors for successful ERP implementation: Exploratory findings from four case studies. Comput Ind. 2005;56(6):529-544.

62. Pinto JK, Slevin DP. Critical factors in successful project implementation. IEEE Trans Eng Manag. 1987;(1):22-27.

63. Kumar V, Maheshwari B, Kumar U. An investigation of critical management issues in ERP implementation: emperical evidence from Canadian organizations. Technovation. 2003;23(10):793-807.

64. Molla A, Loukis I. Success and failure of ERP technology transfer: A framework for analysing congruence of host and system cultures. University of Manchester. Institute for development policy and management (IDPM); 2005

65. Heeks R. Understanding e-governance for development. Institute for Development Policy and Management Manchester; 2001.

66. Walsham G. Making a world of difference: IT in a global context. John Wiley \& Sons, Inc.; 2001.

67. Tjan AK. Finally, a way to put your Internet portfolio in order. Harv Bus Rev. 2001;79(2):76-85.

68. Goodhue DL, Thompson RL. Task-technology fit and individual performance. MIS Q. 1995;213-236.

69. Goodhue Dale L. Development and Measurement Validity of a Task - Technology Fit Instrument for User Evaluations of Information System. Decis Sci. 2007 Jun 7;29(1):105 - 38.

70. Ammenwerth E, Iller C, Mahler C. IT-adoption and the interaction of task, technology and individuals: a fit framework and a case study. BMC Med Inform Decis Mak. 2006;6. 
71. Poon EG, Jha AK, Christino M, Honour MM, Fernandopulle R, Middleton B, et al. Assessing the level of healthcare information technology adoption in the United States: a snapshot. BMC Med Inform Decis Mak. 2006;6.

72. Huang Z, Palvia P. ERP implementation issues in advanced and developing countries. Bus Process Manag J. 2001 Jan 8; 7(3):276-84.

73. Yin RK. Case Study Research : Design and Methods [Internet]. 5th Revised edition. Thousand Oaks, United States: SAGE Publications Inc; 2013 [cited 2016 Aug 22]. 312 p. Available from: http://www.bookdepository.com/Case-Study-Research-Robert-K.-Yin/9781452242569?redirected=true\&utm medi um=Google\&utm_campaign=Base1\&utm_source=AU\&utm_content=Case-Study-Research\&selectCurrency $=$ AU D\&w=AF45AU961BV14DA8ZT47\&pdg=kwd-105542175699:cmp-168747699:adg-9591243219:crv-393781329 39:pid-9781452242569\&gclid=CNqP06mf1M4CFQt-vQodBXMEdA

74. Trochim W, Donnelly JP, Arora K. Research Methods: The Essential Knowledge Base. 2017;

75. Boyatzis RE. Transforming qualitative information: thematic analysis and code development. Sage Publications; 1998. 208 p.

76. Kvale S. InterViews: An Introduction to Qualitative Research Interviewing. 1st ed. Sage Publications, Inc; 1996. 